a protection of 69.1 per cent, but all the oil mixtures averaged 85.9 per cent.

17. Though the trees were retouched, treatment was less effective the second year than the first, the average protection the second year being 7.7 per cent as against 82.1 per cent for the first.

18. Up to a certain age, it appeared that asphalt varies in efficiency inversely as the age of the tree. In other words, the older the trees the less efficient the treatment.

As a result of our investigations at Berryville, we concluded that the most practical manner of handling the borer properly is by worming the tree at the right time every year.

\title{
SUMMARY
}

1. That; the protection offered by white lead is not commensurate with returns.

2. That pruning compound is worthless as a borer protector.

3 . That paper wrappers and wooden veneers, from first observations, appear to be impracticable.

4. That the cost of screening is too great to warrant its use as a borer protector.

5. That the use of asphaltum is attended with injury and that its efficiency as a borer protection is not worthy of the risk to the trees.

6. That worming the trees during the months of August and in early September is the most practicable as well as the cheapest method for the controlling of the borer.

Vice-President G. A. Dean: If there is no discussion, we will now listen to a paper by Mr. George H. Lamson, Jr.

\section{MERCURIAL OINTMENT, AN EFFECTIVE CONTROL OF HEN LICE}

By G. H. Lamson, JR., Storrs, Conn.,

Insecticides, such as the arsenate of lead, lime sulphur, hellebore, hydrocyanic acid gas, carbon bisulphide and nicotine solutions, are of known value and it is from these that the economic entomologist usually draws for the direct control of insects.

To most of you the problems of the control of insects affecting the products of the orchard, field, forest and garden are of most interest, though from time to time your attention is directed to those animal parasites that are of much economic importance. 
Those attempting the control of lice on poultry have become impressed with the difficulty attending this operation for the reason that these small active forms increase in such numbers as to make many types of control impracticable or only partially efficient.

For a long time powders and dust baths have been recommended to those who would free their poultry from lice but it is the ineffectiveness of this method that I would like to emphasize at this time.

To determine the relative value of various means of control, one hundred and fifty well grown white leghorn pullets and male birds were used in the tests made a year ago last summer. This stock was badly infested with lice and they were placed in separate coops and runs so that they would keep under control and at the same time have sufficient exercise. Pyrethrum, the Lawry or Cornell, powder, lard, vaseline, mercurial ointment normal strength, and mercurial ointment dilutes one to two parts, one to three parts, one to five parts, one to ten parts, were tried, together with the usual check lot, each lot consisting of from ten to twelve birds.

The results from these tests were very marked, showing distinctly the inefficiency of the dusting methods even when the operation was repeated several times, for the test extended over the whole summer. The vaseline and lard were more effective over a longer period than the powders. Of the powders, the Cornell (Lawry) powder made up of two and one-half pounds of plaster of paris, one-fourth pint of crude carbolic acid and three-fourths pint of gasoline, caused the death of many lice soon after the powder was applied. As a demonstration of this, these lice were shaken on a large clean piece of paper. This result seemed a positive evidence of effectiveness, but on examining the birds so dusted a few days afterward the numbers of lice seemed to have been but slightly diminished, making continued applications necessary, yet never quite freeing the hens of the lice.

The fact that a hen dusted with a powder shakes much of that powder out of her feathers soon after the mixture has been applied, accounts for a loss of some of the effectiveness of this method. Most dusts, too, lose their effectiveness long before the next generation of lice is hatched. The dust baths are likely to become non-effective by being covered with feathers and accumulated excrements, making them damp and hard.

The use of the mercurial ointment did not cause the death of the lice so quickly, but within a few days no lice could be found upon the hens and this condition lasted over a period of from eight to sixteen weeks. The mercurial ointment caused some burns but the dilution of one part mercurial ointment to two parts vaseline controlled the lice and caused only a few slight irritations that lasted but a short 
time. The more dilute mercurial preparations lost their effectiveness as the amount of mercury was diminished.

After this one hundred and four chickens were treated in the laboratory where they could be under close observation. Here tests were made with these preparations, particularly the mercurial ointments of various strength. The ointment was applied to different parts of the body such as on the head, under the wings and under the anus. The application of the mercurial ointment diluted to the strength of one part to two parts of vaseline was most effective for the largest number of birds when applied under the anus or vent. This was probably due to the fact that the two species of body lice that were more numerous upon birds studied laid their eggs in this region. The application was nearly as effective when placed under the wings and least effective when placed only on the back of the head and neck though for the hens affected with the head and body lice it would give the best results to apply to this region as well as under the anus.

The method of application was to take an amount about the size of a pea and rub it into the base of the feathers. This adheres to the finer portion of the hen's covering and remains there for a long time. A hen thus treated was kept in a glass case for a time under observation; many dead lice were found on the floor of the case while but three live lice were seen to leave her.

Some little confusion occurs in the use of the term mercurial ointment and blue ointment. Mercurial ointment contains 50 per cent of metallic mercury with 50 per cent petrolatum (vaseline).

Blue ointment is a mixture consisting of 67 per cent mercurial ointment and 33 per cent vaseline, and, therefore, contains about 33 per cent of mercury.

Mercurial ointment costs $\$ 1.75$ per pound while blue ointment costs $\$ 1.50$ per pound. It is, therefore, cheaper to buy the mercurial ointment and dilute it with vaseline, using one part of the former to two of the latter. To mix this it is best to place the ingredients on a pane of glass and work them together with a case knife, being sure that the mixing is thoroughly done. Place in a receptacle and label. An ounce of this ointment is sufficient to treat from sixty to seventy hens while the application can be made almost as fast as the hens can be caught. In that much time is likely to be spent in catching the fowl, it is best to do this work after the birds have gone to roost. The cost involved to buy and apply this ointment is less than one-half cent for each hen. To be sure this should not be used for the setting hens on account of the effect it might have upon the respiration of the chick embryo, but if applied one or two months before, the hens will not be 
lousy at the incubating period. For the young chickens a little sweet oil is a practical control measure owing to the tenderness of the skin.

Mercurial ointment is by no means a new insecticide as it has been used for years in the control of Pediculidæ. It has been used also in Denmark and other European countries on cows for cattle lice. Its value as an insecticide for hen lice has as yet not been widely known, yet those who have given this a trial have become enthusiastic over the results obtained and consider it a specific.

It seems hardly possible that a small amount of mercurial ointment applied to a restricted region of a hen's body will free it from lice but the results are above expectation.

Vice-President G. A. Dean: The paper is now open for discussion.

Mr. D. M. Rogers: May I ask how frequently the lice nust be treated?

MR. G. H. LAmson, JR.: We determined that the application in the surnmer would last two or three months. When applied in the fall or winter, it would last four or five months, so I believe in the North that three applications would keep hens free from lice throughout the year. Probably in the South it would take four or five more.

Mr. Z. P. Metcalf: In North Carolina, six applications a year are necessary to keep the hen louse in control. The head louse seems to require more than six applications a year as it is not as easily controlled as the hen louse.

Vice-President G. A. Dean: We will now pass to the next paper by Mr. Z. P. Metcalf.

\section{LIME AS AN INSECTICIDE ${ }^{1}$}

\section{Z. P. Metcalf}

In $1912 \mathrm{Mr}$. C. B. Williams, chief of the Division of Agronomy of our station called my attention to the ravages of the common and four-spotted cow-pea weevils (Bruchus chinensis Linn. and B. quadrimaculatus Fabr.) in the cow peas that were being saved for seed by the station. I at once suggested to him that we fumigate them with carbon bisulphide, which was done, using four pounds to 1,000 cubit feet of air space in a very tight bin at $74^{\circ} \mathrm{F}$. for 72 hours. Contrary to our expectations, however, the cow pea weevils continued to breed in undiminished numbers, although a great many adults were killed at the time the fumigating was done

${ }^{1}$ Contributions from the Department of Zoölogy and Entomology of the North Carolina College of Agriculture and Experiment Station, No. 7. 